\title{
UTILIZZARE RISORSE GRAMMATICALI DELL'ITALIANO PER RIDURRE L'ANSIA LINGUISTICA: RIFLESSIONI E PROPOSTE OPERATIVE
}

\author{
Riccardo Amorati ${ }^{1}$
}

\section{INTRODUZIONE}

Come osserva Pallotti (1998: 220), un'esperienza comune nell'apprendimento di una lingua straniera consiste nel dover affrontare situazioni comunicative nelle quali ci si sente linguisticamente inadeguati. La paura di esporsi, la sensazione di inadeguatezza percepita in relazione ad una performance linguistica, il timore del giudizio del nostro interlocutore, di altri studenti e del docente nella classe di lingua sono alcuni dei fattori che possono essere legati a blocchi psicologici e che possono portare - come conseguenza ultima - alla disistima di sé e all'insuccesso nel processo di apprendimento (Gkonou, Daubney, Dewaele, 2017; Horwitz, 2001; Chen, Chang, 2004, si veda sezione 2).

Nonostante l'ansia linguistica sia un'esperienza comune che interessa la gran parte dei parlanti di lingue seconde (Campbell, Ortiz, 1991: 59; Gkonou et al., 2017), la ricerca glottodidattica in Italia non ha dato largo spazio a questo fenomeno. Pertanto, questo contributo intende fornire una breve visione d'insieme della ricerca sull'ansia linguistica, delineandone le cause e le conseguenze. Poiché la letteratura sull'ansia linguistica ha spesso un taglio teorico piuttosto che pratico (si veda discussione in Amorati, Venturin, 2021), verranno presentate alcune riflessioni pratiche su come è possibile ridurne gli effetti nella classe di italiano L2 per mezzo del nostro agire in classe come docenti, nonché di attività didattiche mirate. In particolare, si discuterà di come alcune risorse linguistiche dell'italiano possono essere utilizzate come punto di partenza per ottenere informazioni sull'ansia linguistica dei nostri studenti e per sensibilizzarsi al fenomeno, si forniranno alcuni spunti operativi e si presenterà - in maniera più dettagliata - un esempio di progetto didattico.

\section{QUADRO TEORICO}

\subsection{L'ansia lingustica: lo stato dell'arte}

Horwitz et al. (1986: 12) definiscono l'ansia linguistica come «un complesso distinto di auto-percezioni, credenze, emozioni e comportamenti legati all'apprendimento delle lingue in classe derivante dall'unicità del processo di apprendimento delle lingue». La qualificazione «linguistica» sottolinea che questo fenomeno è specifico all'uso linguistico. In altre parole, gli

\footnotetext{
${ }^{1}$ University of Melbourne.
} 
(C) Italiano LinguaDue 2. 2021. R. Amorati, Utilizzare risorse grammaticali dellitaliano per ridurre l'ansia linguistica: riflessioni e proposte operative

individui che sperimentano questo tipo di ansia non sono necessariamente soggetti ansiosi in altri aspetti del loro vivere quotidiano. Come sottolinea Horwitz (1986, 2017), questo fenomeno deriva dal fatto che l'apprendimento linguistico si differenzia da altri tipi di apprendimento: imparare una lingua implica allontanarsi dalla propria zona di comfort e trovarsi in una situazione in cui, almeno a livelli di competenza iniziali, si è incapaci di esprimersi in maniera articolata e ci si ritrova ad arrancare mentre si cerca di pronunciare nuovi suoni e di formulare frasi che sarebbero considerate semplici nella propria L1. In questo senso, l'ansia linguistica è tipica di situazioni in cui si percepisce disagio a dare un'immagine di sé inautentica attraverso l'uso della L2. Questo porta alla formazione di quello che Şimşek e Dörnyei (2017) chiamano l'anxious self (sé ansioso), un'immagine di sé stessi in situazioni che causano ansia e che può costituire un deterrente al prosieguo degli studi linguistici. Il sé ansioso, infatti, può contribuire ad alzare il "muro" del filtro affettivo, che - secondo l'ipotesi di Krashen (1986) - deve invece essere abbassato per consentire all'input linguistico comprensibile di essere appreso.

Come osservato prima, l'ansia linguistica è un fenomeno comune. Già agli inizi degli anni '90, Campbell e Ortiz (1991: 59) avanzavano l'ipotesi che almeno la metà degli apprendenti di lingue sperimentassero livelli debilitanti di ansia legati alla loro performance in lingua straniera.

La letteratura in materia ha distinto due tipi di ansia: debilitante e facilitante (si veda, ad esempio, Pallotti, 1998). La prima, come suggerisce il nome, è un'ansia «negativa», che porta ad una riduzione delle capacità del singolo e che si configura come ostacolo. La seconda, invece, si riferisce a quello stato di eccitazione emotiva che però contribuisce a migliorare l'attenzione dell'individuo e la sua performance. Nonostante entrambi i tipi di stati ansiosi siano stati indagati in relazione all'apprendimento linguistico, Horwitz (2017) suggerisce che l'ansia linguistica debba sempre essere concettualizzata come debilitante, in quanto associata a stati emotivi che possono avere un impatto negativo sul piacere dell'apprendere. Per questo motivo, è opportuno, come suggerisce la studiosa, trovare strategie per identificarne le cause e ridurne gli effetti.

\subsubsection{Cause}

La Figura 1 fornisce una panoramica delle cause più comunemente associate all'ansia debilitante che abbiamo identificato sulla base dell'analisi della letteratura (si vedano anche Gregersen, MacIntyre, 2014; Amorati, Venturin, 2021).

Figura 1. Cause dell'ansia linguistica

\begin{tabular}{|c|c|}
\hline $\begin{array}{l}\text { Cause principali } \\
\text { dell'ansia linguistica }\end{array}$ & $\begin{array}{l}\text { - } \begin{array}{l}\text { Pratiche di insegnamento e dinamiche in classe } \\
\text { (Young, 1991) }\end{array} \\
\text { - } \quad \text { Idee controproducenti sugli obiettivi di apprendimento } \\
\text { (Young, 1991; Horwitz, 1988) } \\
\text { - Paura di commettere errori, della correzione e della valutazione } \\
\text { (Gregersen, 2003) } \\
\text { - Sottostima delle proprie abilità } \\
\text { (MacIntyre et al., 1997; Onwuegbuzie et al., 1999) }\end{array}$ \\
\hline
\end{tabular}


(C) Italiano LinguaDue 2. 2021. R. Amorati, Utilizzare risorse grammaticali dellitaliano per ridurre l'ansia linguistica: riflessioni e proposte operative

L'ansia linguistica viene classificata, nella letteratura glottodidattica in lingua italiana, come parte dei fattori affettivi "interni" che influenzano l'apprendimento delle lingue (si veda, ad esempio, Pallotti, 1998). Come sottolineano vari studiosi (si veda, ad esempio, MacIntyre, 2017), l'ansia linguistica è anche uno stato socialmente costruito, ovvero legato al contesto in cui si innesca e sviluppa. Le pratiche di insegnamento e le dinamiche in classe possono essere percepite come particolarmente ansiogene. Young (1990) ha osservato che la comunicazione orale causa maggiore ansietà, probabilmente per l'impossibilità di poter pianificare per tempo quello che verrà detto in uno scambio comunicativo e per la difficoltà aggiuntiva della pronuncia. Pratiche didattiche autoritarie e troppo incentrate sulle valutazione nonché esperienze di apprendimento linguistico negative nel passato sono spesso associate a maggiore ansietà (Dewaele, Magdalena, Saito, 2019; Dewaele, Witney, Saito, Dewaele, 2018; Dewaele, Al-Saraj, 2013; Bailey, 1983).

Gli studenti ansiosi possono anche avere convinzioni controproducenti sull'apprendimento delle lingue. Una tendenza molto comune di molti apprendenti di lingue - specie se di indole perfezionista (si veda, ad esempio, Dewaele, 2017) - è quella di aspettarsi di non commettere errori e di avere pieno controllo delle forme linguistiche apprese. Spesso poi un livello di competenza molto prossimo a quello del parlante nativo viene percepito come l'obiettivo ultimo da raggiungere. Queste ambizioni si scontrano con la realtà: sappiamo che l'errore è un processo naturale della formazione delle interlingua (Selinker, 1972). Sappiamo anche che l'acquisizione di una competenza nativa è quasi impossibile dopo la fase del periodo critico (Lennenberg, 1967). Queste aspettative disattese possono creare un senso di frustrazione e disistima verso le proprie capacità, sfociando in ansia linguistica.

La paura di commettere errori nella L2 rappresenta un altro ostacolo notevole che può creare disagio negli apprendenti. L'errore viene percepito non come un processo naturale dell'apprendimento linguistico, ma piuttosto come un fallimento o come fonte di possibile critica da parte di altri studenti e dell'insegnante (Stöber, Joormann, 2001). Varie ricerche hanno anche dimostrato che l'ansia linguistica spesso è legata a una sottostima delle proprie capacità. Per esempio, gli studenti possono essere convinti di commettere più errori di quelli che effettivamente producono e si demotivano e temono il giudizio altrui in fase di valutazione - la paura di commettere errori può portarli ad un blocco emotivo e impedire una performance in linea con le proprie capacità effettive (Gregersen, MacIntyre, 2014; Onwuegbuzie, Bailey, Daley, 1999; Philipps, 1992).

\subsubsection{Effetti}

Oltre alle conseguenze fisiche dell'ansia linguistica sugli apprendenti (es. senso di tensione, sudorazione, aumento del battito cardiaco), sono stati identificati tre principali effetti di questo fenomeno (Gregersen, MacIntyre, 2014):

- linguistici/cognitivi: influenza sulla performance comunicativa nella L2;

- sociali/interpersonali: propensione a comportamenti che portano ad evitare il rischio, al fine di proteggere la propria immagine sociale;

- emozionali/affettivi: effetto negativo sulla fiducia nelle proprie capacità e sulla sicurezza in sé stessi.

Dal punto di vista linguistico-cognitivo, la letteratura è concorde nel rilevare che l'ansia linguistica debilitante ha un impatto negativo sul successo nell'apprendimento, in quanto rallenta l'acquisizione (MacIntyre, Gardner, 1994; Horwitz, 2000). Questo è dovuto al fatto 
(C) Italiano LinguaDue 2. 2021. R. Amorati, Utilizzare risorse grammaticali dellitaliano per ridurre l'ansia linguistica: riflessioni e proposte operative

che, come confermato da vari studi, uno stato ansioso implica una minore ritenzione delle informazioni e anche maggiori difficoltà nella fase di produzione (si vedano, ad esempio, Horwitz, 2001; Young, 1991).

Dal punto di vista sociale/interpersonale, un chiaro effetto dell'ansia linguistica è la paura di parlare, di essere giudicati da altri e di essere corretti dall'insegnante (si veda ad esempio Horwitz et al., 1986). Questo può portare gli studenti a sviluppare strategie per proteggere la propria immagine sociale. Ad esempio, gli studenti possono rimanere in silenzio od essere meno inclini a rispondere alle domande dell'insegnante, possono dare risposte monosillabiche e cercano di evitare il più possibile interazioni sociali nella L2 (Horwitz et al., 1986).

Dal punto di vista emozionale e affettivo, vari studi hanno dimostrato - come sottolineato in precedenza - che l'ansia linguistica può portare a ridurre la sicurezza in sé stessi e a sottostimare le proprie capacità (Gregersen, MacIntyre, 2014; Phillips, 1992).

\subsection{Suggerimenti pratici per ridurne gli effetti}

Se nella sezione precedente abbiamo individuato le notevoli conseguenze dell'ansia linguistica, qui ci soffermiamo su che cosa possiamo fare, in classe, per attenuare questo fenomeno.

La Tabella che segue presenta una visione d'insieme di strategie che possono essere messe in atto per combattere l'ansia linguistica:

Tabella 1. Strategie per contrastare l'ansia linguistica, riportati in Horwitz. (2017). Commenti sviluppati dall'autore

\begin{tabular}{|c|c|}
\hline Strategie per combattere l'ansia linguistica & Suggerimenti \\
\hline a) la creazione di sistemi di supporto efficaci & Dialogo aperto tra insegnanti e studenti, \\
\hline $\begin{array}{l}\text { b) la promozione di un ambiente di } \\
\text { apprendimento positivo e di supporto }\end{array}$ & $\begin{array}{l}\text { lavoro sul gruppo classe come comunità di } \\
\text { apprendimento. }\end{array}$ \\
\hline c) creazione di aspettative realistiche & $\begin{array}{l}\text { Chiarire obiettivi a breve e lungo termine per gli } \\
\text { studenti e far presente che "sapere" una lingua } \\
\text { non significa avere un accento nativo ed essere } \\
\text { in grado di esprimersi come ci si esprime nella } \\
\text { L1, ma "saper fare" con la lingua, ovvero essere } \\
\text { in grado di compiere atti comunicativi (Balboni, } \\
2008,2012 \text { ) }\end{array}$ \\
\hline $\begin{array}{l}\text { d) preferenza per attività a piccoli gruppi } \\
\text { rispetto ad attività che pongono l'attenzione } \\
\text { sul singolo. }\end{array}$ & $\begin{array}{l}\text { Promuovere una didattica per progetti (Efstratia, } \\
\text { 2014; Moss, Van Duzer, 1998). }\end{array}$ \\
\hline $\begin{array}{l}\text { e) correzione sensibile degli errori e } \\
\text { normalizzazione dell'errore come parte del } \\
\text { processo di apprendimento }\end{array}$ & $\begin{array}{l}\text { Concentrarsi più sulla comunicazione che sulla } \\
\text { forma, utilizzare forme di correzione sensibile. } \\
\text { Ad esempio, l'insegnante ripete la forma corretta } \\
\text { senza interrompere lo scambio comunicativo. }\end{array}$ \\
\hline
\end{tabular}


(C) Italiano LinguaDue 2. 2021. R. Amorati, Utilizzare risorse grammaticali dellitaliano per ridurre l'ansia linguistica: riflessioni e proposte operative

L'obiettivo ultimo dell'implementazione di queste strategie è quello di creare quello che Dörnyei (2018) chiama un «safe-speaking environment», ovvero un ambiente dove gli studenti si trovino a loro agio e si sentano di contribuire senza temere il giudizio altrui. L'importanza di creare un gruppo classe coeso non è ovviamente solo importante per ridurre l'ansia linguistica, ma è alla base di una didattica sensibile alle esigenze e necessità psicologiche del singolo nel processo di apprendimento, in linea con l'approccio comunicativo all'insegnamento della lingua straniera (si veda, ad esempio, Spada, 2007).

Rubio-Alcalá (2017) suggerisce che esistono due mezzi per combattere l'ansia linguistica.

Un modo diretto è di implementare attività specifiche che si focalizzino su questo aspetto psicologico. Un esempio può essere una giornata di sensibilizzazione alla tematica dell'ansia linguistica, per esempio attraverso workshop o interventi di esperti.

Un altro mezzo è quello indiretto: come sottolinea Rubio-Alcalá (2017: 208), «Many activites can be designed to learn the language and, at the same time, reduce anxiety and empower self-esteem. These can be implemented occasionally, when the topics of the lesson and the self-esteem activity are related, or as part of the program».

Il suggerimento di Rubio-Alcalá è in linea con la proposta di Hadfield e Dörnyei (2013) di una serie di attività come parte di quello che definiscono un «programma motivazionale» per l'apprendimento linguistico (si veda anche Amorati, 2020). Queste attività perseguono sia obiettivi linguistici - ovvero servono al consolidamento di forme grammaticali e temi affrontati normalmente nelle classi di lingue - sia obiettivi motivazionali - cioè mirano a rendere consapevoli gli studenti delle loro motivazioni e del loro apprendimento. Come si osserverà nel paragrafo successivo, simili attività possono essere proposte anche in relazione all'ansia linguistica.

3. Risorse gRAMmATICALI DELL'itALIANO CHE SI PRESTANO AD ATTIVITÀ SULL'ANSIA LINGUISTICA

In base all'analisi della letteratura, si ritiene che uno dei mezzi più efficaci per superare l'ansia linguistica sia quello di rendere gli studenti consapevoli del fenomeno, discutere e normalizzare situazioni che creano ansia nell'apprendimento linguistico e lavorare sulla creazione di un gruppo classe inclusivo.

Come osservato in precedenza, questi obiettivi possono essere perseguiti attraverso la promozione di strategie in qualche modo "indirette", ovvero attraverso l'inclusione curricolare di attività utili ai nostri programmi di lingua e anche psicologicamente rilevanti. Si è deciso quindi di scandagliare la grammatica italiana alla ricerca di argomenti che si prestino ad attività atte a generare discussioni in classe sull'ansia linguistica.

Nella Tabella 2 vengono riportati alcuni argomenti grammaticali, divisi nelle due categorie "elementare" e "intermedio-avanzato". Non si distingue tra livelli intermedi e avanzati in quanto i temi grammaticali affrontati ai livelli avanzati sono spesso un consolidamento di forme apprese ai livelli intermedi (ad esempio il congiuntivo). 
(C) Italiano LinguaDue 2. 2021. R. Amorati, Utilizzare risorse grammaticali dellitaliano per ridurre l'ansia linguistica: riflessioni e proposte operative

Tabella 2. Alcuni esempi di argomenti grammaticali per discussioni sull'ansia linguistica

\begin{tabular}{|l|l|}
\hline \multicolumn{1}{|c|}{ Livello } & \multicolumn{1}{c|}{ Argomenti che si prestano all'implementazione } \\
\hline Elementare & $\begin{array}{l}\text { Argomenti associati all'espressione di emozioni } \\
\text { Verbi riflessivi (sentirsi) e verbi di emozione, come ad esempio espressioni } \\
\text { idiomatiche con avere («avere paura»). } \\
\text { Imperfetto con verbi di emozione, stati d'animo e pensiero }\end{array}$ \\
& $\begin{array}{l}\text { Altri argomenti rilevanti } \\
\text { Aggettivi e concordanza di genere e numero } \\
\text { Imperativo informale } \\
\text { Verbi modali }\end{array}$ \\
Condizionale dei verbi modali per suggerimenti \\
\hline Intermedio e \\
avanzato & $\begin{array}{l}\text { Argomenti associati all'espressione di emozioni } \\
\text { Congiuntivo o forma «di }+ \text { infinito» con verbi che indicano emozioni, dubbi, } \\
\text { ipotesi , desideri, etc. }\end{array}$ \\
& $\begin{array}{l}\text { Altri argomenti rilevanti } \\
\text { Comparativi e superlativi } \\
\text { Condizionale passato dopo verbi del pensare (oltre che del dire) al passato } \\
\text { Si impersonale e passivante } \\
\text { Verbi causativi (far fare - far sentire) }\end{array}$ \\
\hline
\end{tabular}

Come si può osservare, la tabella contiene sia argomenti direttamente legati a stati emotivi sia argomenti non "emotivi" ma utili per attività di sensibilizzazione sull'ansia linguistica. Con il termine "argomento emotivo", ci si riferisce in questa sede a contenuti grammaticali che marcano stati emotivi. L'italiano è una lingua per cui esistono forme grammaticali che sono tipicamente associate a stati emotivi e mentali. Questo significa che quando si affrontano queste forme grammaticali, si possono presentare attività relative all'ansia linguistica.

Nei paragrafi che seguono si offrono alcuni spunti didattici per livello di attività che possono essere inserite all'interno di una pianificazione curricolare per il consolidamento di regole grammaticali affrontate in classe.

\section{- Livelli elementari}

Come si può vedere in Tabella 2, un tema grammaticale associato alle emozioni ai livelli elementari è costituito dai verbi riflessivi, che spesso sono utilizzati per marcare stati d'animo. Pensiamo, ad esempio, alle forme mi sento..., mi preoccupo, mi innervosisco, mi vergogno, ecc. Allo stesso modo, l'imperfetto viene generalmente utilizzato per marcare stati d'animo al passato, nonché, tra altri usi, anche per descrivere abitudini nel passato (ero preoccupato, mi sentivo agitato, etc.). Gli esercizi di pratica su questo tempo verbale possono quindi essere utilizzati per far riflettere gli studenti su aspetti relativi ai propri stati emotivi all'inizio o in fasi specifiche del processo di apprendimento e su come sono cambiati nel tempo.

Altri argomenti grammaticali possono costituire un target linguistico in attività che mirano a far riflettere gli studenti sull'ansia linguistica. Ai livelli elementari, ad esempio, si può chiedere agli studenti di utilizzare aggettivi di emozione e di esprimere coerentemente 
(C) Italiano LinguaDue 2. 2021. R. Amorati, Utilizzare risorse grammaticali dellitaliano per ridurre l'ansia linguistica: riflessioni e proposte operative

l'accordo con il soggetto (In classe mi sento preoccupato/preoccupata, quando...). Si può chiedere poi agli studenti di dare consigli su come essere un migliore apprendente di lingue utilizzando l'imperativo, sia in forma affermativa che negativa, o di utilizzare i verbi modali per esprimere volontà, probabilità, desiderio, etc. (Se voglio essere più sicuro di me..., posso provare a parlare di più, etc.).

\section{- Livelli intermedio-avanzati}

Per i livelli intermedio-avanzati, il congiuntivo e la forma $d i+$ infinito nelle subordinate sono gli argomenti più tipicamente associati ad asserzioni relative alla speranza, alle emozioni, ai dubbi, alle paure. Possono quindi essere sfruttati per chiedere agli studenti di condividere opinioni e dubbi sull'apprendimento delle lingue e sulle loro emozioni relative all'uso linguistico sia nel presente (Penso che imparare una lingua sia difficile / Temo di aver paura a parlare, ecc.) sia nel passato (Quando ho iniziato a studiare l'italiano pensavo fosse/ sarebbe stato...., e invece...").

Altri argomenti rilevanti possono essere i comparativi. Si può chiedere agli studenti, ad esempio, di utilizzare vari aggettivi di emozione per descrivere come si sentono in vari momenti del loro apprendimento (ad esempio quando parlano in classe, quando usano la lingua in contesti non istituzionali, etc.). Il si impersonale e passivante possono essere utilizzati, alla stregua dell'imperativo, per fare generalizzazioni su sensazioni comuni condivise da apprendenti di lingue e per formulare "massime" relative a come migliorare (Quando si studia una lingua, (non) si deve...; Quando ci si sente a disagio nell'usare la lingua, (non) si deve...). Infine, i verbi causativi - ed espressioni come mi/ci fa sentire... - possono essere utilizzati in classe per far riflettere gli studenti sulle emozioni negative - ma anche positive - associate all'apprendimento delle lingue e all'uso delle lingue.

\section{Un esempio}

Si presenta qui un esempio di attività "modello" che può essere calibrata per tutti i livelli e che include alcune delle risorse grammaticali mostrate in precedenza. Nella presentazione dell'attività si distinguono titolo/scopi/dinamica e commento, seguendo il modello di presentazione utilizzato da Balboni (2012). Si rimanda il lettore/la lettrice alla sezione 4 per la presentazione più dettagliata di un progetto didattico a sé stante che include alcune di queste attività e che offre esempi concreti di esercizi pratici che possono essere proposti in classe.

Titolo:

Pensieri, stati d'animo ed emozioni relativi all'apprendimento linguistico.

Scopi:

Linguistici (presente e/o passato prossimo con gli ausiliari essere e avere di verbi riflessivi che indicano emozioni, imperativo, si passivante, si impersonale, congiuntivo) e psicologici (creare consapevolezza sull'ansia linguistica, discutere degli stati emotivi più comuni associati all'apprendimento linguistico, sviluppare strategie per superarla).

Fase 1

a) Domande per tutti $i$ livelli

- Come ti senti quando parli in classe in italiano?

- Hai mai avuto paura di parlare in classe? Quando? 
(C) Italiano LinguaDue 2. 2021. R. Amorati, Utilizzare risorse grammaticali dellitaliano per ridurre l'ansia linguistica: riflessioni e proposte operative

- Come ti senti quando parli l'italiano?

- Come ti sentivi quando hai iniziato a studiarlo?

- Ti sei mai scoraggiato quando hai incontrato un ostacolo nello studio dell'italiano?

- Quali emozioni dovrebbe provare un buon studente di lingue secondo te?

b) Domande aggiuntive per i livelli intermedio-avanzati

- Cosa pensi dell'apprendimento di una lingua? Crea frasi usando verbi come pensare, temere, credere

- Di cosa hai paura/Cosa temi quando parli in italiano in classe?

Fase 2

Dai 5 consigli/ordini a un apprendente di lingua usando l'imperativo, sulla base delle risposte che hai ricevuto (livelli elementari), e il si impersonale/passivante (livelli elementari-intermedi).

Dinamica e commento:

L'attività serve per sensibilizzare gli studenti ai più comuni stati emotivi che si provano nel corso dell'apprendimento linguistico. Agli studenti può essere chiesto di inserirli in uno spazio online che consente la creazione di wordcloud. In questo modo, gli stati emotivi più comuni emersi possono essere mostrati visivamente a tutta la classe. Questo può costituire un punto di partenza per espandere il lessico sugli aggettivi e può essere un modo per iniziare una discussione sul ruolo che le emozioni hanno sull'apprendimento linguistico.

\section{UN ESEMPIO DI PROGETTO DIDATTICO}

In questa sezione, forniamo un esempio di progetto didattico che può essere proposto in classe. Il progetto è una versione riveduta e ampliata di due attività presentate in un recente articolo sul tema dell'ansia linguistica che non aveva un focus specifico sull'italiano L2 ma che si rifaceva ad esempi in lingua inglese (Amorati, Venturin, 2021). In una delle attività veniva introdotto il film Inside Out nella fase "motivazionale". In questa sede, l'idea viene rivista e sviluppata ulteriormente per rendere il film un tema centrale del progetto. Nella descrizione sintetica dell'attività si pone l'accento sul livello di riferimento, gli scopi, le aree linguistiche che possono essere incluse e la dinamica dell'attività. Al termine, si presenta anche un commento critico.

L'attività è strutturata tenendo in considerazione le caratteristiche chiave dell'apprendimento per progetto, un approccio in base al quale la lingua e il contenuto vengono acquisiti mentre gli studenti lavorano su compiti complessi che portano alla creazione di un prodotto finale (Efstratia, 2014; Moss, Van Duzer, 1998). L'apprendimento basato su progetti è riconosciuto come un approccio efficace per l'acquisizione sia delle abilità linguistiche che di una varietà di altre abilità interpersonali, di problem solving e di pensiero critico (si veda, ad esempio, Stoller, 2006). 
(C) Italiano LinguaDue 2. 2021. R. Amorati, Utilizzare risorse grammaticali dellitaliano per ridurre l'ansia linguistica: riflessioni e proposte operative

- Visione d'insieme dell'attività

Tabella 3. Fasi e durata dell'attività

\begin{tabular}{|c|c|}
\hline Fasi dell'attività & Durata suggerita \\
\hline $\begin{array}{l}\text { Fase 1: } \\
\text { Fase motivazionale iniziale, brainstorming in } \\
\text { classe del tema e acquisizione di nuovi contenuti } \\
\text { relativi alle emozioni. }\end{array}$ & 1 o 2 lezioni. \\
\hline $\begin{array}{l}\text { Fase 2: } \\
\text { Creare una risorsa per apprendenti di lingue. }\end{array}$ & $\begin{array}{l}2 \text { lezioni (durata variabile a seconda del numero } \\
\text { di temi proposti e dello scopo dell'attività - } \\
\text { consolidamento di regole già viste vs } \\
\text { presentazione di nuovi contenuti grammaticali } \\
\text { attraverso la sequenza globalità - analisi - } \\
\text { sintesi). }\end{array}$ \\
\hline $\begin{array}{l}\text { Fase 3: } \\
\text { Discussione in classe su temi emersi. }\end{array}$ & 1 lezione. \\
\hline Fase 4: Testo riflessivo da consegnare & $\begin{array}{l}\text { A casa o da svolgere in classe come prova di } \\
\text { verifica. }\end{array}$ \\
\hline
\end{tabular}

- Livello

Tutti i livelli, a seconda degli argomenti.

- $\quad$ copi

a) Linguistici (si veda Tabella 2 per l'inclusione di prompt nella fase 2 dell'attività). Ad esempio:

- dare suggerimenti (imperativo e verbi modali al condizionale);

- descrivere le emozioni (usando opportune concordanze e verbi riflessivi);

- descrivere le esperienze passate (uso dell'imperfetto);

- esprimere opinioni, dubbi (congiuntivo);

- riflessione e sviluppo della propria competenza glottomatetica (tutti i livelli).

b) Psicologici: creare consapevolezza sull'apprendimento linguistico e i suoi ostacoli, cercare di contrastare l'ansia linguistica sensibilizzando gli studenti al fenomeno e lavorando sulla creazione di una comunità di apprendimento inclusiva e coesa.

\section{- Metodologie didattiche}

L'insegnamento avviene in italiano utilizzando un approccio comunicativo che ha come obiettivo l'acquisizione di competenza comunicativa, intesa come insieme di conoscenze astratte sulla lingua e abilità concrete relative all'uso linguistico (si veda Balboni, 2012). Questo modello di competenza comunicativa pone l'accento non solo sul "sapere la lingua", ma anche sulla capacità di utilizzare le proprie competenze per comprendere, produrre e 
(C) Italiano LinguaDue 2. 2021. R. Amorati, Utilizzare risorse grammaticali dellitaliano per ridurre l'ansia linguistica: riflessioni e proposte operative

manipolare testi ("saper fare"), contribuendo così ad eventi comunicativi governati da regole sociali, pragmatiche e culturali ("saper fare con la lingua"). L'insegnamento avviene attraverso l'esposizione ad input autentico e diversificato che viene analizzato in modo induttivo, riorganizzato e riprodotto. La presentazione di contenuti testuali/mediali avviene seguendo le tipiche fasi dell'unità didattica delineate da Balboni (2008, 2012):

- motivazione (ipotesi e previsioni sui temi presentati per attivare conoscenze pregresse e stimolare la curiosità degli studenti);

- globalità (attività di skimming e scanning per riassumere i punti centrali dei contenuti presentati e confermare o meno le ipotesi formulate in precedenza) e - se rilevante ai fini degli obiettivi didattici - analisi (lavoro sul materiale presentato per scoprire in modo induttivo regole grammaticali, testuali etc.);

- pratica e sintesi (applicazione, fissazione e reimpiego delle regole e conoscenze apprese).

In linea con approcci pedagogici correnti (si vedano Balboni, 2008, 2012; Spada, 2007), si propongono attività comunicative (presentazioni degli studenti, lavoro a gruppi, didattica per progetti) che incoraggiano gli studenti a mettere in pratica le proprie competenze linguistiche per comunicare con gli altri e/o per produrre un prodotto finale tangibile.

\section{- Dinamica e commento}

Fase 1: Diventiamo esperti o esperte di emozioni

Nella fase "motivazione" si presenta agli studenti il trailer del film Inside Out in lingua italiana ${ }^{2}$. Il film racconta la storia di una giovane ragazza e delle cinque emozioni primarie che popolano la sua mente. L'obiettivo è di sensibilizzarli alla tematica "emozioni e stati d'animo" per mezzo di un'attività stimolante e che attiva conoscenze pregresse.

Gli studenti guardano la locandina e provano a intuire di cosa parli il film. Poi, dopo aver visto il trailer, nella fase globalità si chiede agli studenti di rispondere alle seguenti domande:

1. Quale storia viene raccontata nel trailer?

2. In che modo viene descritta la mente della protagonista?

3. Qual è la funzione di ognuno dei personaggi rappresentati?

Dopo un'iniziale discussione in plenum, agli studenti vengono mostrati i seguenti prompt, che li invitano ad osservare l'immagine presentata in Figura 3 e a utilizzare lessico relativo all'espressione emozionale.

\section{Quali emozioni sono rappresentate in Figura 3?}

2. Quali tra queste emozioni sono rilevanti per te o per persone che conosci nell'apprendimento di una lingua? Prova a fare un brainstorming di alcune situazioni comuni e poi completa la tabella in Figura 4.

\footnotetext{
2 Si veda: https://www.youtube.com/watch?v=pI1PY1dxdYs.
} 
(C) Italiano LinguaDue 2. 2021. R. Amorati, Utilizzare risorse grammaticali dell'italiano per ridurre l'ansia linguistica: riflessioni e proposte operative

Figura 3. Le emozioni del film "Inside out" Tratto da:

https://lavocedidentro.wordpress.com/2015/10/19/inside-out-emozionicercasi/
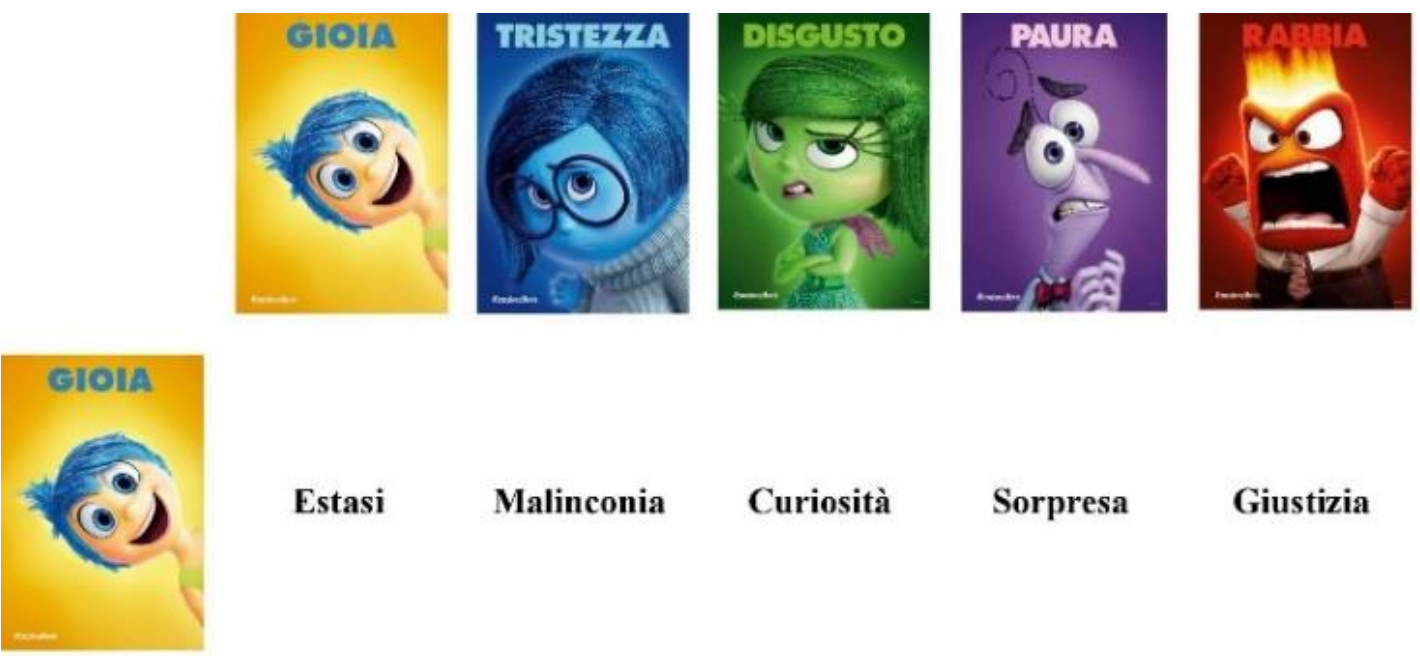

\section{Estasi}

Malinconia

Curiosità

Sorpresa

Giustizia

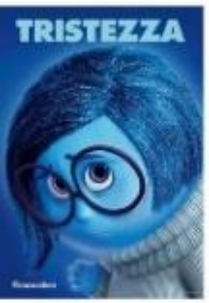

Malinconia

Disperazione

Disprezzo di sè

Ansia

Tradimento

DISCUSTO

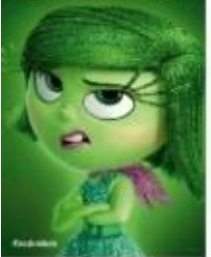

\section{PAURA}
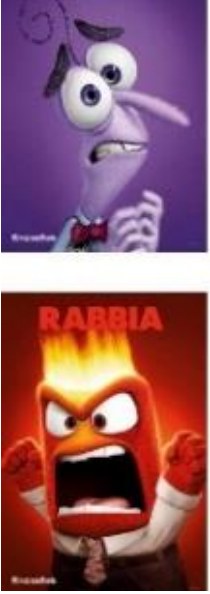

\section{Curiosità}

Disprezzo di sè

Pregiudizio

Repulsione

Disprezzo
Ansia

Sorpresa

Giustizia
Disprezzo
Odio

Ira 
(C) Italiano LinguaDue 2. 2021. R. Amorati, Utilizzare risorse grammaticali dell'italiano per ridurre l'ansia linguistica: riflessioni e proposte operative

Figura 4. Mappare le emorioni in diversi ambiti di uso linguistico

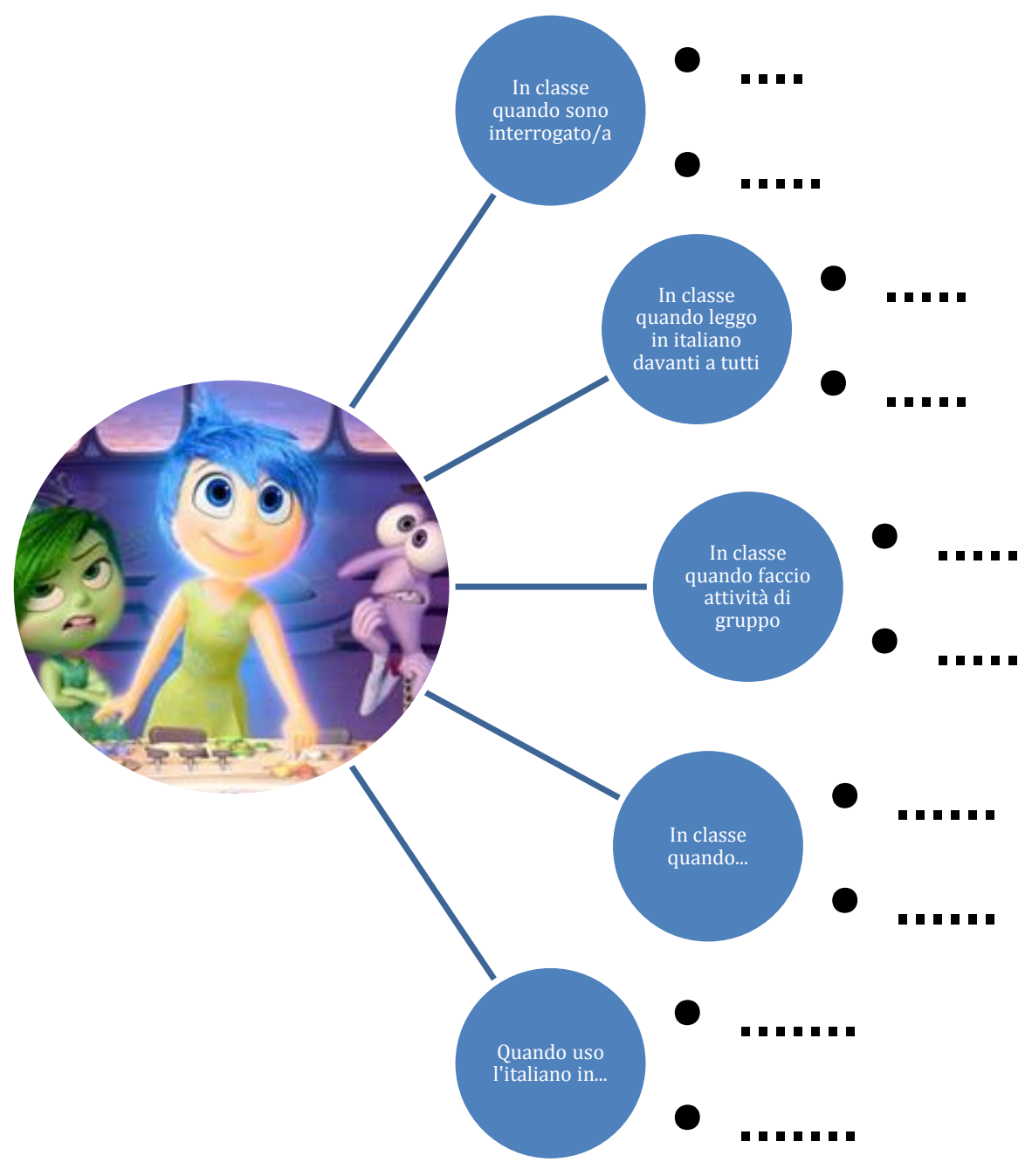

Lo scopo di questo esercizio è di aumentare la consapevolezza degli studenti sulla rilevanza delle emozioni nell'apprendimento delle lingue e di aiutarli a "mappare" quali emozioni sono rilevanti in determinate situazioni. Al termine dell'attività, si fa una discussione in plenum: quali contesti/situazioni sono più spesso associati alla "Paura"? Quali alla "Gioia"? Si chiede poi agli studenti di spiegare quale potrebbe essere la causa della frequenza di determinate emozioni in alcuni contesti.

$\mathrm{Al}$ termine di questa prima parte dell'attività viene proposta la lettura di un testo reperibile online sulle emozioni primarie: Inside Out: come migliorare la nostra vita a partire dalle 5 emozioni primarie (Albè, 2015). Il testo tratta in maniera approfondita il ruolo delle emozioni e serve ad abbattere alcuni preconcetti sulla valenza negativa di alcuni stati emotivi. Nel testo si osserva, per esempio, come la paura non sia sempre negativa, ma anzi sia un'emozione che serve a "proteggere". Nella fase motivazione, viene chiesto agli studenti di provare a intuire il contenuto del testo leggendo solo il titolo. Gli studenti lavorano a gruppi e provano a indovinare alcuni dei consigli che potrebbero essere contenuti nell'articolo. Poi il testo viene 
(C) Italiano LinguaDue 2. 2021. R. Amorati, Utilizzare risorse grammaticali dell'italiano per ridurre l'ansia linguistica: riflessioni e proposte operative

letto in classe. Come parte della fase di "globalità", si chiede agli studenti di rispondere ad alcune domande di skimming e scanning, ad esempio:

1. Quali sono le emozioni primarie secondo il testo?

2. Come si chiama lo studioso che ha fatto ricerca sulle emozioni?

3. Cosa intende l'autrice del testo quando dice che le 5 emozioni primarie non sono di per sé positive o negative? Quali esempi vengono fatti?

4. In che modo la tristezza, la paura e la rabbia possono essere positive?

5. Quali suggerimenti dà l'autrice per migliorare la nostra vita a partire dalle emozioni?

È possibile poi presentare successive attività come parte della fase di "analisi” (Balboni, 2008, 2012) e "pratica". Ad esempio, agli studenti può essere richiesto di analizzare alcune caratteristiche del linguaggio giornalistico o di identificare alcune forme grammaticali ricorrenti nel testo e poi di metterle in pratica attraverso appositi esercizi di fissazione e reimpiego.

\section{Fase 2: Applichiamo le nostre conoscenze per creare una risorsa online}

Agli studenti viene spiegato che lo scopo della seconda fase dell'attività è di creare una risorsa online per studenti di lingue. Questa risorsa serve ad aumentare la consapevolezza sul processo di apprendimento delle lingue, a identificare alcuni ostacoli che gli studenti di lingue incontrano e a formulare strategie per superarli. Agli studenti viene fornito uno spazio condiviso online (ad esempio, una pagina Padlet) e una serie di riquadri con prompt prestabiliti ed alcuni esempi. Di seguito sono riportati alcuni esempi di prompt e modelli che si basano a grandi linee sulle attività proposte in lingua inglese da Amorati, Venturin (2021) e che sono specificatamente adattati alla classe di italiano L2.

Per ricollegarsi al tema centrale del film Inside Out, gli studenti devono riflettere sulle emozioni primarie nella mente degli apprendenti di lingue (Figura 5) e sui loro ricordi come apprendenti (Figura 6) al fine di produrre un prodotto finale utile per gli studenti di lingue.

Figura 5. Il quartiere generale della mente degli apprendenti di lingue https://www.paulekman.com/blog/the-science-of-inside-out/

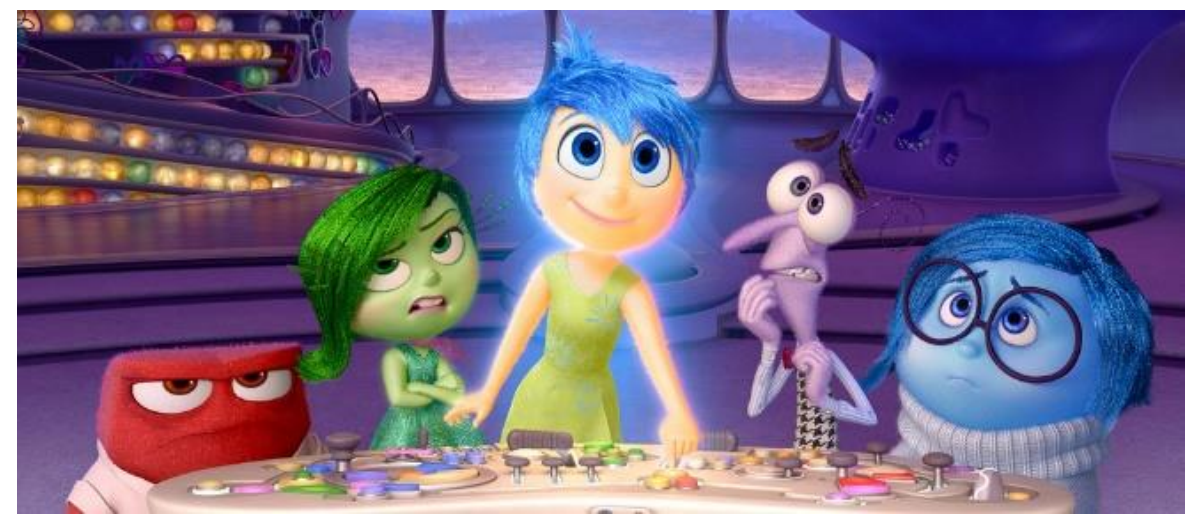


(C) Italiano LinguaDue 2. 2021. R. Amorati, Utilizzare risorse grammaticali dellitaliano per ridurre l'ansia linguistica: riflessioni e proposte operative

Figura 6. Gli scaffali dei ricordi e delle emozioni

https://www.slashfilm.com/inside-out-easter-eggs/

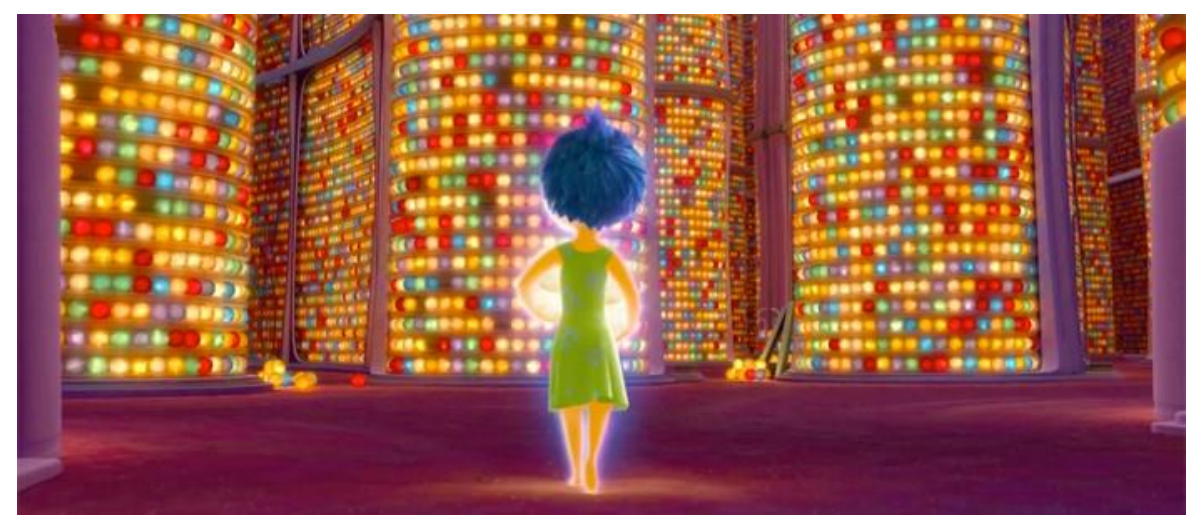

Tema 1: Quando parlo una lingua, mi sento...

Struttura: verbi di emozioni e aggettivi che esprimono emozioni, con opportune concordanze di genere e numero:

- Emozioni positive, ad esempio, felice, intelligente, esperto, ecc.

- Emozioni negative, ad esempio, frustrato, preoccupato, ecc.

- Emozioni neutre, ad esempio,...

Tema 2: Quando ho iniziato ad imparare l'italiano, mi sentivo... Ora mi sento... Pensate a qualcosa che è cambiato - o in meglio o in peggio.

Struttura: imperfetto per azioni ripetute nel passato; congiuntivo o condizionale retto da verbi del pensare e del dire al passato, per esempio:

- Quando ho iniziato a imparare l'italiano, mi sentivo spaesato. Facevo spesso errori e mi preoccupavo. Ora ho sempre un po' paura ma mi sento meno preoccupato.

- Quando ho iniziato a imparare l'italiano pensavo che avrei potuto parlare come un(a) madrelingua. Ora ho capito che il mio accento è un elemento che mi identifica e di cui andare fiero.

Tema 3: Come dev'essere lo stato emotivo dell'apprendente di lingue ideale? Scrivi alcune «regole» per gli studenti di lingue

Struttura: imperativo in forma affermativa e negativa, per esempio:

- Accetta di buon grado il rischio! Impara dai tuoi errori e dagli altri!

- Conosci il proverbio italiano «sbagliando si impara»? Non avere paura di sbagliare, anche i migliori sbagliano.

- Non temere di avere una cattiva pronuncia. Ricorda che spesso gli accenti stranieri susci tano curiosità.

- Non avere paura del giudizio degli insegnanti. Ricorda che loro sanno bene cosa significa imparare una lingua straniera. 
(C) Italiano LinguaDue 2. 2021. R. Amorati, Utilizzare risorse grammaticali dell'italiano per ridurre l'ansia linguistica: riflessioni e proposte operative

Tema 4: Suggerimenti per gli studenti di lingue

Struttura: verbi modali al condizionale, per esempio:

- Dovresti provare a parlare in classe, anche se non sei completamente sicuro della forma grammaticale della tua frase.

- Potresti...

Tema 5: Ambiti su cui vorrei suggerimenti (aggiungi all'elenco e offri suggerimenti ai tuoi compagni di classe utilizzando "dovere" al condizionale). Puoi inserire problemi personali o riferirti a problemi generici che spesso incontrano gli studenti di lingue, per esempio:

- Cosa devo fare per sentirmi meno preoccupato di parlare in classe? $\rightarrow$ Dovresti pensare che nessuno è lì per giudicare! Tutti commettono errori, compresi i madrelingua!

Tema 6: Dubbi emorioni e stati d'animo nell'apprendimento linguistico.

Utilizza verbi che esprimono dubbi, opinioni, volontà ed emozioni per esprimere le tue opinioni relative all'apprendimento linguistico. Non scordarti di usare il congiuntivo.

- Penso che imparare una lingua richieda molti sforzi.

- Ho paura che le persone mi giudichino quando sentono il mio accento.

Come si può vedere, la maggior parte dei suggerimenti mira anche alle strutture linguistiche, e può quindi servire a consolidarle (fase "pratica" nella sequenza di Balboni, 2008, 2012). È possibile però anche utilizzarle per presentare nuovo materiale didattico (fase "analisi"). In quel caso, agli studenti si può chiedere di sottolineare nel testo le forme, classificarle in tabelle e astrarre in modo induttivo la regola. Si può poi chiedere loro di applicarla in altri contesti attraverso esercizi mirati.

La durata di quest'attività dipende dal numero di temi che gli insegnanti decidono di proporre. All'inizio di ogni lezione, gli studenti sono divisi in piccoli gruppi (3-4) e lavorano su uno dei temi per un determinato periodo di tempo (ad esempio 15 minuti). Ogni gruppo lavora a turni su un tema diverso e contribuisce in modo collettivo ad aggiornare lo spazio online. Se richiesto dalla consegna (si veda il Tema 5), i gruppi devono fornire suggerimenti ad altri studenti. $\mathrm{Al}$ termine dell'attività avremo uno spazio online che contiene una rassegna di emozioni tipiche associate all'apprendimento linguistico, riflessioni sul significato di "apprendere una lingua", ma anche suggerimenti - formulati dagli studenti stessi - su come superare paure e ansie legate all'apprendimento. La risorsa può essere periodicamente aggiornata da tutta la classe.

\section{Fase 3 e 4: Discussione in classe e consegna di un diario riflessivo}

Agli studenti viene chiesto di scrivere un breve testo nella L2 in cui riflettono sull'attività. In questo diario critico finale, agli studenti viene chiesto di basarsi sui contenuti del Padlet e di scrivere un testo riflessivo sulla loro esperienza come studenti di lingue. Devono poi indicare che cosa hanno imparato attraverso questa attività, riferendosi anche alle esperienze dei compagni e delle compagne. In caso si decida che questa riflessione sia oggetto di valutazione, si può chiedere agli studenti di inserire nei loro testi alcune strutture linguistiche incontrate nell'attività (ad esempio: "Nella tua riflessione inserisci almeno 5 verbi al congiuntivo, 2 periodi ipotetici, 3 forme di imperativo con pronomi, etc.). 
(C) Italiano LinguaDue 2. 2021. R. Amorati, Utilizzare risorse grammaticali dell'italiano per ridurre l'ansia linguistica: riflessioni e proposte operative

\section{CONCLUSIONI}

Questo contributo si è posto l'obiettivo di fornire una panoramica sul fenomeno dell'ansia linguistica e delle sue principali cause ed effetti, nonché di fornire proposte operative per diminuirne gli effetti in classe attraverso attività che perseguono obiettivi linguistici e psicologici (Rubio-Alcalá, 2017; Hadfield, Dörnyei, 2014) e che sfruttano il fatto che l'italiano codifichi grammaticalmente stati emotivi. Alla luce della scarsità di ricerche sul tema con un focus pratico - ovvero con l'obiettivo di fornire attività concrete che possono essere facilmente implementate in classe - si ritiene che studi come quello attuale siano necessari per contribuire ad un dialogo virtuoso tra ricercatori nel settore dell'educazione linguistica e docenti. In future ricerche, sarà importante analizzare come attività di sensibilizzazione al tema dell'ansia linguistica vengano percepite in classe e se contribuiscano effettivamente a un miglioramento dello stato emotivo degli studenti. Un altro filone di ricerca che si ritiene promettente riguarda l'analisi di come i manuali e i sillabi dei corsi per l'insegnamento dell'italiano a stranieri includano sezioni di riflessione su aspetti affettivi ed emozionali relativi all'apprendimento linguistico (ad esempio ansia, motivazione, etc.): prevedere una riflessione su questi fenomeni è importante nelle nostre classi, visto l'impatto di questi fattori sul processo di apprendimento.

\section{RIFERIMENTI BIBLIOGRAFICI}

Albè M. (2015), "Inside Out: come migliorare la nostra vita a partire dalle 5 emozioni primarie", in Green Me: https://www.greenme.it/vivere/costume-e-societa/inside-outemozioni-primarie/

Amorati R. (2020), "Drawing upon disciplinary knowledge to foster long-term motivation: Implementing future L2 selves in the Australian tertiary L2 classroom", in Fornasiero J., Reed S. M. A., Amery R., Bouvet E., Enomoto K., Xu H. L. (eds.), Intersections in language planning and policy, vol. XXIII, Springer, Cham, pp. 335-351:

https://doi.org/10.1007/978-3-030-50925-5_21.

Amorati R., Venturin B. (2021), “'I command thee thou shalt speak': Understanding and counteracting foreign language anxiety in the classroom", in Journal of Theories and Research in Education, XVI, 3, pp. 39-57: https://doi.org/10.6092/issn.1970$2221 / 12350$.

Bailey K. M. (1983), "Competitiveness and anxiety in adult second language learning: Looking at and through the diary studies", in Seliger H. W., Long M. H. (eds.), Classroom-Oriented Research in Second Language Acquisition, Newbury House, Rowley, pp. 67-103.

Balboni P. (2008), Le sfide di Babele. Insegnare le lingue nelle società complesse, UTET, TorinoNovara.

Balboni P. (2012), Fare educazione linguistica. Insegnare italiano, lingue straniere e lingue classiche, UTET, Torino-Novara.

Campbell C. M., Ortiz J. A. (1991), "Helping students overcome foreign language anxiety: A foreign language anxiety workshop", in Horwitz E. K., Young, D. J. (eds.), Language anxiety: From theory and research to classroom implications, Prentice-Hall, Englewood Cliffs, pp. 153-168. 
(C) Italiano LinguaDue 2. 2021. R. Amorati, Utilizzare risorse grammaticali dell'italiano per ridurre l'ansia linguistica: riflessioni e proposte operative

Chen T.-Y., Chang G. B. Y. (2004), "The relationship between foreign language anxiety and learning difficulties", in Foreign Language Annals, XXXVII, 2, pp. 279-89.

Dewaele J. M. (2017), "Psychological dimensions and foreign language anxiety", in Loewen S., Sato M. (eds.), The Routledge handbook of instructed second language acquisition, Routledge, London, pp. 433-450.

Dewaele J. M., Al-Saraj T. M. (2013), "Foreign Language Anxiety: Some conceptual and methodological issues", in Journal of Psychology, LXVIII, 3, pp. 71-78.

Dewaele J.-M., Witney J., Saito K., Dewaele L. (2018), "Foreign language enjoyment and anxiety: The effect of teacher and learner variables", in Language Teaching Research, XXII, 6, pp. 676-697: 10.1177/1362168817692161.

Dewaele J. M., Magdalena A. F., Saito K. (2019), "The effect of perception of teacher characteristics on Spanish EFL Learners' anxiety and enjoyment", in The Modern Language Journal, CIII, 2, pp. 412-427: 10.1111/modl.12555.

Dörnyei Z. (2018), Safe-speaking environment, 52nd Annual International IATEFL* Conference and Exhibition, Brighton, 9th to 13th April 2018: https:/ /www.youtube.com/watch?v=-wDmI-DUt-c.

Efstratia D. (2014), "Experiential education through project-based learning", in Procedia Social and Behavioral Sciences, CLII, 7, pp. 1256-1260.

Gkonou C., Daubney M., Dewaele J. M. (eds.) (2017), New insights into language anxiety: theory, research and educational implications, Multilingual Matters, New York.

Gregersen T. S. (2003), "To err is human: A reminder to teachers of language-anxious students", in Foreign Language Annals, XXXVI, 1, pp. 25-32:

https://doi.org/10.1111/j.1944-9720.2003.tb01929.x.

Gregersen T., MacIntyre P. D. (2014), Capitalizing on individual differences: From premise to practice, Multilingual Matters, Bristol.

Hadfield J., Dörnyei Z. (2013), Motivating learning, London, Routledge.

Horwitz E.K. (1988), “The beliefs about language learning of beginning university foreign language students", in The Modern Language Journal, LXXII, 3, pp. 283-294:

https://doi.org/10.1111/j.1540-4781.1988.tb04190.x.

Horwitz E. K. (2000), “It ain't over til it's over: On foreign language anxiety, first language deficits, and the confounding of variables", in The Modern Language Journal, LXXXIV, 2 , pp. 256-259.

Horwitz E. (2001), "Language anxiety and achievement", in Annual Review of Applied Linguistics, XXI, pp. 112-126: https://doi.org/10.1017/S0267190501000071.

Horwitz E. K., Horwitz M. B., Cope J. (eds.) (1986), "Foreign Language Classroom Anxiety", in Modern Language Journal, LXX, 2, pp. 125-32.

Horwitz E. K. (2017), "On the misreading of Horwitz, Horwitz, and Cope (1986) and the need to balance anxiety research and the experiences of anxious language learners", in Gkonou C., Daubney M., Dewaele J. M. (eds.), New insights into language anxiety: theory, research and educational implications, Multilingual Matters, Bristol, pp. 31-47.

Krashen S. D. (1986), Principles and practice in second language acquisition, Pergamon Press, Oxford. Lenneberg E. H. (1967), Biological foundations of language, John Wiley \& Sons, New York.

MacIntyre P. D., Gardner R. C. (1994), "The subtle effects of language anxiety on cognitive processing in the second language", in Language Learning, XLIV, 2, pp. 283305: https://doi.org/10.1111/j.1467-1770.1994.tb01103.x.

MacIntyre P. D., Noels K. A., Clement R. (1997), "Biases in self-ratings of second language proficiency: the role of language anxiety", in Language Learning, XLVII, 2, pp. 256-287. 
(C) Italiano LinguaDue 2. 2021. R. Amorati, Utilizzare risorse grammaticali dellitaliano per ridurre l'ansia linguistica: riflessioni e proposte operative

MacIntyre P. D. (2017), "An overview of language anxiety research and trends in its development", in Gkonou C., Daubney M., Dewaele J. M. (eds.), New insights into language anxiety: theory, research and educational implications, Multilingual Matters, Bristol, pp. 11-30.

Moss D., Van Duzer C. (1998), Project-based learning for adult English language learners, National Center for ESL Literacy Education, Washington.

Onwuegbuzie A. J., Bailey P., Daley C. E. (1999), "Relationship between anxiety and achievement at three stages of learning a foreign language", in Perceptual and Motor Skills, LXXXVIII, 3, pp. 1085-1093.

Pallotti G. (1998), La seconda lingua, Bompiani, Milano.

Philipps E. M. (1992), "The effects of language anxiety on students' oral test performance and attitudes", in The Modern Language Journal, LXXVI, 2, 14-26: 10.1111/j.15404781.1992.tb02573.x.

Rubio-Alcalá F. D. (2017), "The links between self-esteem and language anxiety and implications for the classroom", in Gkonou C., Daubney M., Dewaele J. M. (eds.), New insights into language anxiety: theory, research and educational implications, Multilingual Matters, Bristol, pp. 198-216.

Selinker L. (1972), "Interlanguage”, in International Review of Applied Linguistics, X, 3, pp. 21931: http://dx.doi.org/10.1515/iral.1972.10.1-4.209.

Şimşek E., Dörnyei Z. (2017), “Anxiety and L2 self-images: The 'anxious self”, in Gkonou C., Daubney M., Dewaele J. M. (eds.), New insights into language anxiety: theory, research and educational implications, Multilingual Matters, Bristol, pp. 51-69.

Spada N. (2007), “Communicative Language Teaching”, in Cummins J., Davison C. (eds.) International Handbook of English Language Teaching. Springer International Handbooks of Education, vol. XV, Springer, Boston: https://doi.org/10.1007/978-0-387-463018_20.

Stöber J., Joormann J. (2001), "Worry, procrastination, and perfectionism: Differentiating amount of worry, pathological worry, anxiety, and depression", in Cognitive Therapy and Research, XXV, 1, pp. 49-60: 10.1023/A:1026474715384.

Stoller F. (2006), "Establishing a theoretical foundation for project-based learning in second and foreign language contexts", in Beckett G. H., Miller P. C. (eds.), Project-based second and foreign language education: Past, present, and future, Information Age, Greenwich, pp. 1940.

Young D. J. (1990), “An investigation of students' perspectives on anxiety and speaking”, in Foreign Language Annals, XXIII, 6, pp. 539-553: https://doi.org/10.1111/j.19449720.1990.tb00424.x.

Young D. J. (1991), "Creating a low-anxiety classroom environment: What does language anxiety research suggest?", in The Modern Language Journal, LXXV, 4, pp. 426-439: 10.1111/j.1540-4781.1991.tb05378.x. 